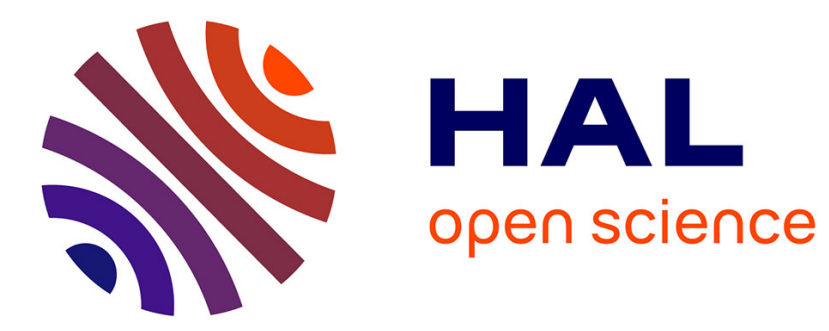

\title{
Le pilotage de la performance éducative : expérience innovante et acte manqué
}

Chaker Haouet, Larbi Hasrouri, Dany Deschamps

\section{To cite this version:}

Chaker Haouet, Larbi Hasrouri, Dany Deschamps. Le pilotage de la performance éducative : expérience innovante et acte manqué. Audit Comptabilité Contrôle: Recherches Appliquées, 2020, ํ8 (2), pp.67. 10.3917/accra.008.0067 . hal-03354508

\section{HAL Id: hal-03354508 \\ https://hal.science/hal-03354508}

Submitted on 28 Sep 2021

HAL is a multi-disciplinary open access archive for the deposit and dissemination of scientific research documents, whether they are published or not. The documents may come from teaching and research institutions in France or abroad, or from public or private research centers.
L'archive ouverte pluridisciplinaire HAL, est destinée au dépôt et à la diffusion de documents scientifiques de niveau recherche, publiés ou non, émanant des établissements d'enseignement et de recherche français ou étrangers, des laboratoires publics ou privés. 


\title{
LE PILOTAGE DE LA PERFORMANCE ÉDUCATIVE : EXPÉRIENCE INNOVANTE ET ACTE MANQUÉ
}

\author{
Chaker Haouet, Larbi Hasrouri, Dany Deschamps
}

Association Francophone de Comptabilité | «ACCRA »

$2020 / 2 \mathrm{~N}^{\circ} 8 \mid$ pages 67 à 87

ISSN 2617-2399

DOI 10.3917/accra.008.0067

Article disponible en ligne à l'adresse :

https://www.cairn.info/revue-accra-2020-2-page-67.htm

Distribution électronique Cairn.info pour Association Francophone de Comptabilité.

(C) Association Francophone de Comptabilité. Tous droits réservés pour tous pays.

La reproduction ou représentation de cet article, notamment par photocopie, n'est autorisée que dans les limites des conditions générales d'utilisation du site ou, le cas échéant, des conditions générales de la licence souscrite par votre établissement. Toute autre reproduction ou représentation, en tout ou partie, sous quelque forme et de quelque manière que ce soit, est interdite sauf accord préalable et écrit de l'éditeur, en dehors des cas prévus par la législation en vigueur en France. Il est précisé que son stockage dans une base de données est également interdit. 


\section{Le pilotage de la performance éducative : expérience innovante et acte manqué}

Educational performance management: innovative experience and a missed act

\section{Chaker Haouet}

Laboratoire VALLOREM - Université d'Orléans

Maître de Conférences en Sciences de Gestion

chaker.haouet@univ-orleans.fr

\section{Larbi Hasrouri}

Laboratoire VALLOREM - Université d'Orléans

Professeur Agrégé d'Economie-Gestion, Doctorant en Sciences de Gestion

hasrouri@free.fr

\section{Dany Deschamps}

Inspecteur d'Académie

Délégué académique à la Formation des Personnels de l'Éducation Nationale

dany.deschamps@gmail.com

\section{RÉSUMÉ}

Cette étude analyse comment la diffusion d'un outil de pilotage de la performance est perturbée dans sa mise en œuvre par l'interaction des logiques gestionnaire et politique. Cette question est abordée en prenant appui sur une expérimentation portée par un Rectorat d'Académie française. L'étude entend contribuer aux travaux sur les facteurs perturbant la mise en œuure d'outils de pilotage de la performance dans un contexte organisationnel particulier qui est celui des Établissements Publics Locaux d'Enseignement (EPLE). Ce travail met en avant que les rationalités différentes, sous-jacentes à l'interaction des logiques gestionnaire et politique, peuvent conduire à perturber le développement des outils de pilotage de la performance au sein des organisations publiques.

MOTS CLÉS - expérience innovante, OVARO, outil de pilotage, pilotage de la performance, processus. 


\section{ABSTRACT}

This study analyzes how the diffusion of a performance management tool is disrupted in its implementation by the interaction of managerial and political logic. This question is addressed by relying on an experiment carried out by a Rectorate of the French Academy. The study intends to contribute to work on the factors disrupting the implementation of performance management tools in a particular organizational context which is that of the Local Public Teaching Establishments (EPLE). This work highlights that the different rationalities, underlying the interaction of managerial and political logic, can lead to disrupt the development of performance management tools within public organizations.

KEYWORDS - innovative experience, OVAR ${ }^{\odot}$, management tool, performance management, process.

\section{Introduction}

Au cours des deux dernières décennies, les outils de gestion, et plus particulièrement ceux du contrôle de gestion, se sont progressivement répandus dans de nombreuses organisations évoluant dans la sphère publique (Dreveton 2014 ; Bollecker 2016). En France, leur diffusion au sein de ces organisations s'est faite le plus souvent sous la contrainte de leur environnement institutionnel (Eyraud 2013 ; Dreveton 2014 ; Bollecker 2016) au travers de lois et de textes réglementaires (la LOLF, la LRU, la MAP, etc.) $)^{1}$. Les différentes réformes voulues par les Pouvoirs Publics ont amené les organisations publiques à transformer leur mode de management qui fait du contrôle de gestion un dispositif central de celui-ci (Berland et De Rongé 2016).

Avec le déploiement des outils de gestion et des systèmes de pilotage de la performance, une nouvelle logique gestionnaire s'est progressivement diffusée dans ces organisations, « où préexistent de fortes logiques institutionnelles » (Boitier et Rivière 2016, p. 48). Toutes ces réformes sont influencées par les thèses et les politiques de ce qu'il est convenu d'appeler le Nouveau Management Public (en anglais: NPM pour New Public Management). Comme le souligne Eyraud (2013, p. 194), le « socle commun [à ces réformes] réside dans le fait de considérer que l'État et ses entités doivent rendre des comptes,

1. La Loi Organique relative aux Lois de Finances (LOLF) a été votée en 2001. La Loi relative aux Responsabilités et Libertés des Universités (LRU) a été votée en 2007. La Modernisation des Actions Publiques (MAP) date de 2012. 
faire preuve de leur performance et améliorer cette performance, d'où I'usage généralisé d'indicateurs ». C'est ainsi que la mise en application des principes du NPM s'est imposée, et que les réformes portées par ce dernier ont contraint les organisations publiques à " intégrer au cœur de [leur] management des outils de gestion qui véhiculent des valeurs initialement associées à la gestion des organisations privées » (Dreveton 2017, p. 11). Parmi ces valeurs, celle de performance occupe désormais une place centrale dans ce nouveau mode management des organisations publiques.

L'introduction des outils de pilotage de la performance au sein des organisations publiques a fait l'objet de plusieurs travaux académiques, qu'il s'agisse de travaux analysant cette introduction ou de travaux révélant les difficultés liées au déploiement des outils du contrôle de gestion (Ducrocq et Gervais 2013 ; Boitier et Rivière 2016) ou encore de travaux sur les échecs de mise en œuvre ces outils (Dreveton et al. 2012 ; Bollecker 2013).

La littérature sur l'innovation pour le pilotage de la performance s'est surtout intéressée aux déterminants organisationnels ou à ceux liés à l'environnement (Moynihan 2006 ; Walker 2006 ; Damanpour et Schneider 2008 ; Carassus et al. 2014). D'autres travaux se sont intéressés à une catégorie de déterminants parmi lesquels le rôle et les caractéristiques de managers et des leaders politiques et administratifs (Gould-Williams 2004 ; Damanpour et Schneider 2008 ; Bollecker 2016). Si ces travaux ont permis de mettre en évidence l'influence différenciée de ces déterminants sur la dynamique d'innovation dans les organisations publiques, les facteurs freinant le développement des outils de pilotage de la performance ont, à notre connaissance, été peu étudiés (du moins en France), notamment dans le contexte retenu dans cet article. Notre recherche a donc pour objet d'étudier la mise en œuvre d'un outil de pilotage de la performance développé dans un contexte organisationnel particulier qui est celui des Établissements Publics Locaux d'Enseignement (EPLE) relevant d'un Rectorat d'Académie. Notre question de recherche est donc : Comment l'interaction des logiques gestionnaire et politique peut-elle freiner la mise en œuvre d'un outil de pilotage de la performance ? Pour y répondre, l'étude prend appui sur une expérimentation de l'allocation des moyens sur la base du couple « responsabilités-résultats » en identifiant des variables actionnables pour atteindre les objectifs fixés dans le cadre de la LOLF. 
L'article est structuré en trois parties. La première partie présente les enjeux du pilotage de la performance éducative II s'agira dans cette première partie d'examiner successivement le pilotage de la performance éducative par la chaîne de valeur (1.1.) et la problématique du pilotage de cette performance par les indicateurs (1.2.). La deuxième partie analyse le processus de l'expérimentation menée dans un Rectorat d'Académie visant à piloter la performance des EPLE. Cette deuxième partie présentera tour à tour : la méthodologie de la recherche (2.1.) et le contexte de la recherche (2.2.). Enfin, la troisième partie met en évidence et discute les résultats.

\section{Les enjeux du pilotage de la performance éducative}

Le pilotage de la performance a progressivement pénétré le Service Public d'Éducation (Bessire et Fabre 2011) notamment à travers la LOLF. Les indicateurs y occupent une place centrale, comme d'ailleurs dans toute organisation. Ces indicateurs restent cependant majoritairement centrés sur les résultats du processus principal (ou processus " métier $»^{2}$ ). Or, la performance se joue aussi dans le choix des processus « soutien $»^{3}$ et de leur organisation comme dans celui des processus « support $»^{4}$. II s'agira dans cette première partie d'examiner successivement le pilotage de la performance éducative par la chaîne de valeur (1.1.) et la problématique du pilotage de la performance éducative par les indicateurs (1.2.).

\subsection{Le pilotage de la performance éducative par la chaîne de valeur}

La création de la valeur est aujourd'hui en tête des préoccupations de tout responsable, qu'il s'agisse de responsables politiques (Ministre,

2. Pour l'EPLE le processus « métier » est constitué des activités d'enseignement ou d'éducation.

3. Ceux qui rendent possible la mise en œuvre du processus « métier » (exemple, la maintenance informatique des postes et des salles).

4. Ceux qui administrent et gèrent les deux précédents (la direction de l'EPLE ou l'intendance...). 
Recteur, notamment) ou d'acteurs de terrain (les chefs d'établissement, notamment). Elle les contraint à rompre avec les logiques bureaucratiques de cloisonnement par métiers et de coordination par la règle. De fortes pressions sont ainsi exercées sur les acteurs de terrain pour implanter des outils de gestion, et plus particulièrement ceux du contrôle de gestion, visant à transformer leur mode de management. Ce qui en soi constitue une rencontre entre deux valeurs : celle portée traditionnellement par les organisations publiques et celle portée par I'entreprise privée (Eyraud 2013 ; Dreveton 2017).

L'approche de la performance publique par la valeur impose aux organismes concernés de nouvelles exigences en termes organisationnels, gestionnaires et culturels. Les métiers doivent se décloisonner, apprendre à communiquer et à travailler ensemble, notamment pour optimiser leurs interfaces, sources de fréquents dysfonctionnements (Lorino 1999, p. 31). Cet impératif se traduit par un besoin de mobilité des compétences et des personnes, l'expert d'un métier donné devant, par exemple, être prêt à se déplacer d'une structure d'expertise métier à une structure de projet, mode d'organisation qui s'est fortement développé dans ce nouveau contexte. II s'agit de mobiliser les membres d'une organisation, à travers des valeurs communes, de fédérer leurs énergies en vue d'atteindre les objectifs de l'entreprise jugés stratégiques.

Le pilotage de la performance par la chaîne de valeur exige de faire usage des méthodes de pilotage fondées sur la modélisation de l'entreprise en processus transversaux à l'organisation et aux métiers. Le pilotage par processus devient ainsi un outil puissant de changement des modes de gestion et des cultures. À ce titre, il permet de redonner un sens et un contenu concret, quoiqu'instable, à la notion de " performance publique » (Lorino 1999).

La chaîne de valeur montre qu'il est possible d'assembler les processus " métier » avec les processus « support » et " soutien » pour créer de la valeur et être performant. La performance globale dépend en effet de la performance de chaque activité, mais aussi de la performance des liaisons qui existent entre les activités (Porter 1986).

C'est principalement à ces aspects que va s'attacher le paragraphe suivant. 


\subsection{La problématique du pilotage de la performance publique par les indicateurs}

Le pilotage de la performance se traduit par une attention particulière portée sur les processus et activités créatrices de la performance et, ce faisant, sur les liens entre le résultat et les variables qui sont à l'origine de la création de celui-ci. C'est ce que l'on recherche quand on parle de pilotage de la performance. Selmer (2005, p. 39) le définit comme « l'ensemble des pratiques formelles qu'une organisation crée pour aider les acteurs de la performance $»$.

Il est d'usage de décomposer les activités d'une organisation en processus ; celui-ci étant défini comme un ensemble d'activités et de tâches élémentaires concourant à un but commun : fournir un service ou fabriquer un produit. II est alors possible d'opérer une approche processus pour le service public (Lorino 1998). Trois types de processus peuvent être définis : le processus « métier » qui rend le service attendu de l'organisation, les processus « soutien » rendant possible la mise en œuvre du processus « métier » et enfin les processus « support » administrant et gérant les deux précédents. Les trois types de processus sont essentiels à la performance.

Dans le cas de processus concourant à des services publics stabilisés ${ }^{5}$ et standardisés ${ }^{6}$, le pilotage de la performance se borne à une régulation des ressources par les collectivités publiques pourvoyeuses. Dans ce contexte, la gestion des compétences et des carrières (fondée sur une structure de métiers) amène la culture de l'organisation à devenir corporatiste, voire cloisonnée entre métiers.

Face à la massification de l'enseignement (secondaire et supérieur, notamment), des processus stables et standardisables se révèlent peu adaptés. L'État a dû tenir compte de l'hétérogénéité et de la difficulté des publics d'apprenants. Des dispositifs d'Accompagnement Personnalisé (AP) et un Encadrement Éducatif ( $A E$ ) ont été mis en œuvre dans les Collèges et Lycées pour répondre à des besoins de réactivité et de différenciation de la société moderne. Le Service Public d'Éducation (SPE) ne pouvait pas rester standard et stable dans ses processus. II a dû évoluer vers plus de personnalisation et de différenciation, condui-

5. Faciles à mettre en œuvre.

6. Le même service pour tous dans la mesure où il n'est pas personnalisé. 
sant à une certaine innovation?. Cette ambition met en avant l'importance d'un pilotage de la performance innovant visant à répondre aux besoins de processus complexes et personnalisés.

Dans le contexte du SPE, la définition du besoin ne ressort pas seulement du marché, mais elle reflète une volonté générale, relayée, interprétée et explicitée par la puissance publique dans son expression tant nationale (l'État), régionale (les rectorats d'Académie) que locale (les Collectivités). Se trouve posée, malgré tout, la question de la mesure de la performance. La mesure est une nécessité pour rapprocher les résultats des actions aux objectifs fixés. Sans la mesure, on ne sait pas où l'on est et où l'on va. L'expression de la mesure est l'indicateur.

Les indicateurs de résultats constatent des réussites ou des défaillances, mais n'expliquent pas leur provenance. L'origine des défaillances reste difficile à identifier en raison de la difficulté à modéliser les processus à l'œuvre qui sont difficilement standardisables. Ainsi, un même cours peut être dispensé en autant de différentes manières qu'il y a d'enseignants. En outre, si les objectifs sont quantifiés à travers la LOLF, ils ne font pas toujours sens pour les acteurs de terrain. Ainsi, un établissement scolaire sait que sa performance est inférieure au standard attendu, mais il ne peut en expliquer les raisons dans le détail, si tant est qu'on puisse l'expliquer localement.

Lorsqu'un indicateur interpelle que ce soit négativement ou positivement, il convient d'identifier les mécanismes qui le produisent et de repérer les leviers d'actions sur ces mécanismes. En effet, savoir qu'un indicateur est dans le rouge ne donne pas la solution pour en sortir. Une baisse, par exemple, du taux de réussite à un examen peut avoir des dizaines de causes plus ou moins liées à l'action directe des enseignants : stabilité des épreuves et des programmes, déroulement des épreuves, y compris la conception du sujet, déroulement de la formation, ambiance de la classe, etc. Une fois les processus, les acteurs concernés et leurs missions identifiés, il est possible de mettre en place une stratégie pour piloter la performance.

Dans cette première partie, les enjeux du pilotage de la performance éducative ont été présentés ; il convient, à présent, d'aller plus loin,

7. L'article 34 de la loi de 2005 sur l'école prévoit explicitement cette innovation par l'expérimentation dans les établissements scolaires. Cet article a été maintenu par la loi sur l'école suivante (2013). 
c'est-à-dire de nous intéresser à une expérimentation d'un pilotage de la performance dans des EPLE relevant d'un Rectorat d'Académie.

\section{Une étude de cas d'expérimentation d'un pilotage de la performance au sein d'un Rectorat d'Académie}

Dans cette deuxième partie, nous nous penchons sur une expérimentation d'un pilotage de la performance dans des EPLE relevant d'un Rectorat d'Académie. Après avoir présenté la méthodologie sur laquelle s'appuie notre analyse (2.1.), nous abordons le contexte de la recherche en mettant en exergue l'angle d'approche constitué par la méthode $\operatorname{OVAR}^{\circledR}$ (2.2.1), puis l'opérationnalisation de la méthode (2.2.2.) et, enfin, I'abandon de l'expérimentation (2.2.3.).

\subsection{Méthodologie de l’étude}

Cette recherche s'appuie sur une étude de cas au sein d'un Rectorat d'Académie française, que nous baptisons EPPE, ayant expérimenté une allocation des ressources sur la base du couple " responsabilités-résultats » en identifiant des variables actionnables pour atteindre les objectifs fixés dans le cadre de la LOLF. Environ $30 \%$ des EPLE relevant de EPPE étaient concernés par l'expérimentation. En effet, les chefs d'établissement n'étaient pas contraints d'être partie prenante de cette expérimentation ; ils y étaient, cependant, incités (incitation sur l'enveloppe horaire allouée si ces établissements jouaient le jeu). L'expérimentation s'est déroulée sur la période 2011-2014. L'un des trois auteurs de cet article a reconstitué le protocole sa mise en place à partir de sa participation observante.

Notre recherche peut s'apparenter à la recherche-intervention dans la mesure où le chercheur réalise une analyse empirique d'une situation de gestion. Elle s'en distingue toutefois en donnant au chercheur un statut « d'ingénieur » qui conçoit l'outil support de sa recherche, le co-construit, et agit à la fois comme animateur et évaluateur de sa mise en œuvre dans les organisations, contribuant, ce faisant, à l'émergence de représentations et de connaissances scientifiques nouvelles. Ces connaissances scientifiques « visent à fournir un guide à « l'ingénieur 
organisationnel », pour construire des problèmes complexes et piloter des processus ». (Chanal et al. 2015, p. 3).

Comme le recommande Stake (1994), nous adopterons une forme narrative, story telling, afin de pouvoir retranscrire la complexité et la singularité du contexte (Ahrens et Chapman 2006). La démarche adoptée est celle d'une participation observante au sein de l'Académie étudiée durant quatre ans pour un des auteurs, en tant qu'Inspecteur référent à l'évaluation des EPLE. La participation observante se distingue de l'observation participante par une implication en profondeur du chercheur (Lalonde 2013). Cette position a permis de participer à la réflexion sur la mise en place de l'expérimentation, puis de suivre sur une période significative le processus de la mise en œuvre de celle-ci.

\subsection{Contexte de la recherche : une expérimentation d'un outil de pilotage de la performance par la méthode OVAR $^{\circledR}$}

La mise en place de l'outil a été validée par le Recteur de EPPE en tant qu'expérimentation gestionnaire ${ }^{8}$. Sa finalité était d'améliorer l'équité, notamment territoriale entre les EPLE, dans la répartition des moyens. L'objectif principal d'une allocation des ressources sur la base du couple "responsabilités-résultats", pour le Recteur, est de sortir de la logique des moyens et de la reconduction quasi automatique des moyens de l'année précédente pour tendre vers un modèle plus interactif dans lequel les moyens sont en partie alloués en fonction du degré d'atteinte des objectifs négociés.

\subsubsection{La méthode OVAR ${ }^{\circledR}$ comme angle d'approche}

Lors de la réflexion sur la mise en place d'un pilotage de la performance des EPLE, la méthode OVAR ${ }^{\circledR}$ a constitué un angle d'approche. Développée et diffusée en France, dès la fin des années 1980, par des professeurs du groupe HEC, la méthode "Objectifs-Variables d'Action- Responsables » (OVAR) « répond, d'après Löning et Selmer (2011, p. 111), à la préoccupation première de créer davantage de cohérence dans l'entreprise, de faire en sorte d'améliorer la convergence des buts des différents services/départements/entités ». L'idée de réduire une

8. C'est la seule innovation de cette nature dans un Rectorat 
pertinence quelque peu centripète à cause des mini-systèmes épars dans l'Éducation Nationale a été soulignée, il y a déjà plus de dix ans, par un rapport de I'Inspection Générale (2007, p. 15) : « II n'existe pas de système global de contrôle de gestion : absence de définition partagée et de méthodologie commune, approches fragmentées (outils isolés, utilisés à des degrés divers, et propres à chaque structure), des indicateurs de "base" plus que des indicateurs de pilotage qui font système, défaut d'optimisation de la chaîne "objectifs-moyens-résultats" (pas de lien formalisé et mesurable)».

Dans la mesure où la volonté de la tutelle est d'associer les ressources aux objectifs et indicateurs de performance, la méthode OVAR ${ }^{\circledR}$ paraissait un angle d'approche pertinent dans le cadre d'un dialogue de gestion entre les EPLE et les autorités académiques (la tutelle). En ce sens, elle paraissait pouvoir constituer un levier efficace de mobilisation des acteurs au sein des EPLE pour l'amélioration les résultats. Dans sa mise en œuvre pratique, la méthode $O V A R^{\circledR}$ se décompose en trois grandes phases:

- une phase de réflexion sur les missions des entités et de détermination de leurs objectifs (O) opérationnels,

- une phase d'identification de leurs variables d'action (VA),

- une phase d'analyse des responsabilités (R).

Elle peut commencer par une analyse stratégique au niveau de la direction générale qui donnera lieu à la détermination des objectifs de l'organisation et, ce faisant, au déploiement de la stratégie au sein de celle-ci. Mais le plus souvent elle s'inscrit dans une démarche "Bottom-Up » en construisant l'articulation entre les objectifs opérationnels dans le cadre d'axes stratégiques et les plans d'action aux différents niveaux hiérarchiques.

Dans notre cas, les objectifs découlent du contrat d'objectifs négocié entre l'autorité de tutelle (Recteur d'Académie ou Directeur départemental) et le chef d'établissement. Le périmètre de l'évaluation est constitué par l'EPLE. Une auto-évaluation est pratiquée par les parties prenantes de manière collégiale à partir d'un diagnostic global. L'évaluation interne collégiale peut être nourrie par l'évaluation externe de la part des autorités académiques ou des inspections régionales ou départementales. Le diagnostic sert à la politique d'établissement matérialisée par le projet d'établissement co-construit par une ins- 
tance créée ad hoc par la loi de 2005 : le conseil pédagogique. Celui-ci va inscrire les (variables $d^{\prime}$ ) actions dans le projet d'établissement en cohérence avec la stratégie du projet académique. L'EPLE peut décider de ses orientations stratégiques si elles se situent dans les axes stratégiques du projet académique. Nous avons donc bien les objectifs (O) dans le cadre du contrat d'objectifs qui va décliner les variables ou leviers (VA) dans le cadre du projet d'établissement. Des responsables (R) des actions inscrites dans le projet d'établissement vont ensuite rendre compte à travers l'évaluation interne. Le schéma 1 ci-dessous contextualise cette démarche dans le cas d'un EPLE.

\section{Schéma 1 : L’application de la méthode $\mathrm{OVAR}^{\circledR}$ à l'EPLE}

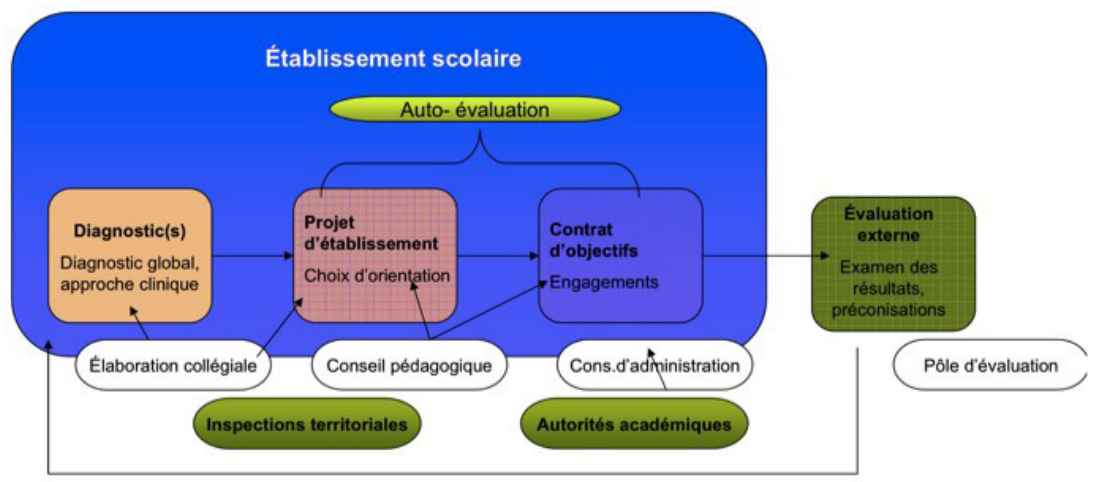

Dans la situation décrite, le processus d'instrumentation vise en réalité l'amélioration de la qualité, mesurée en termes de résultats, du service éducatif rendu aux élèves. Lequel service est censé avoir le caractère d'un objectif général et partagé. L'analyse de la qualité du service fourni par les EPLE est essentielle pour identifier les chaînes de performance et les axes de progrès. Une chaîne de performance part d'un indicateur à améliorer, identifie les activités produisant cet indicateur, décortique le processus pour mettre en évidence les axes de progrès, fixe les objectifs, identifie les variables d'action à travailler, identifie les acteurs concernés et distribue les actions à mener en fonction des responsabilités identifiées. Si, par exemple, le diagnostic fait ressortir des résultats très contrastés entre les différents publics accueillis (indicateur : les résultats en fonction des écoles d'origine) en 
raison de leur hétérogénéité, l'objectif à poursuivre sera de progresser en matière d'équité pour développer la performance de l'établissement auprès des publics les plus en difficulté. L'efficacité ainsi que le coût de chaque action entreprise sont mesurés pour, à terme, identifier les actions les plus pertinentes. Les variables d'action ainsi que les responsabilités retenues sont indiquées dans le tableau 1 ci-dessous.

Tableau 1 : Les variables d'action et responsabilités retenues

\begin{tabular}{|c|c|}
\hline Variables d'action & Responsabilités \\
\hline Composition des classes & $\begin{array}{c}\text { Équipe de direction, services d'orientation, } \\
\text { DSDEN }{ }^{9}\end{array}$ \\
\hline Composition des équipes pédagogiques & $\begin{array}{l}\text { Équipe de direction, conseil pédagogique, } \\
\text { professeurs principaux }\end{array}$ \\
\hline Projets culturels et éducatifs & $\begin{array}{l}\text { Tous les acteurs internes et les élèves avec } \\
\text { les partenaires des projets. }\end{array}$ \\
\hline $\begin{array}{l}\text { Organisation de l'accompagnement } \\
\text { personnalisé }\end{array}$ & $\begin{array}{c}\text { Conseil pédagogique, professeurs, } \\
\text { personnels éducatifs au sens large et les } \\
\text { élèves }\end{array}$ \\
\hline Méthodes didactiques et pédagogiques & $\begin{array}{l}\text { Professeurs, inspecteurs, conseil } \\
\text { pédagogique }\end{array}$ \\
\hline
\end{tabular}

\subsubsection{L'opérationnalisation de la méthode}

Depuis la mise en place des contrats d'objectifs en 2005 découlant de la LOLF, I'institution fonctionne par contractualisation et par projet. Le contrat relève d'une démarche " top down » et le projet plutôt d'une démarche "Bottom up ». Le dialogue de gestion, rendez-vous annuel entre les équipes de direction des EPLE et leur tutelle (Recteur) est le moment privilégié pour mettre en cohérence ces approches et déterminer les moyens affectés aux EPLE pour qu'ils puissent accomplir leurs missions dans le cadre du projet académique et d'établissement (c'est, en quelque sorte, leur feuille de route stratégique).

Un établissement est caractérisé par sa carte des formations. Celle-ci est négociée avec le Rectorat et la Collectivité territoriale de rattachement (Conseil Régional pour les Lycées et Conseil Départemental pour les Collèges). La carte des formations donne la structure de l'établis-

9. Direction des Services Départementaux de l'Éducation Nationale (DSDEN). 
sement : les diplômes préparés, les niveaux de classes, les options ou spécialités ouvertes. Sur la base de cette carte est établie la prévision des effectifs qui servira de base au calcul des moyens en heures. Cette prévision est calculée à partir des données des services d'orientation pour les nouveaux entrants et sortants et à partir des Taux Apparents de Passage (TAP) dans le niveau supérieur pour les autres. À partir des programmes et référentiels en vigueur, les grilles horaires vont permettre de calculer les moyens nécessaires au fonctionnement d'un EPLE. L'ensemble des moyens ainsi déterminés constitue la Dotation Horaire Globalisée $(\mathrm{DHG})^{10}$.

Le classement de l'établissement en fonction de la population scolaire de sa zone de recrutement sera très influent sur sa dotation. Si un Collège, par exemple, est situé en Zone Urbaine Sensible (ZUS), il bénéficiera de moyens supplémentaires pour accomplir sa mission au nom de la solidarité nationale au service de l'équité. Ces moyens permettent entre autres d'améliorer l'indicateur $\mathrm{H} / \mathrm{E}^{11}$ et, d'autre part, d'alléger I'Obligation Réglementaire de Service (ORS) ${ }^{12}$ des enseignants devant la classe pour dégager du temps en accompagnement personnalisé des élèves ou pour prendre en considération la difficulté à enseigner devant certaines classes.

Dans notre cas, le service du contrôle de gestion académique a calculé des DHG théoriques préétablies qui ont été comparées aux DHG réelles obtenues par les établissements. La DHG théorique était calculée à trois niveaux décrits ci-dessous :

- Calcul de la « DHG théorique socle » nommée DHG1 à partir des horaires obligatoires, sans choix de groupe ou d'option, de chacune formations ouvertes dans l'établissement,

10. C'est l'enveloppe budgétaire octroyée par le Rectorat en heures : on distingue les heures postes (HP) et les heures supplémentaires à l'année (HSA) et effectives, c'est-à-dire ponctuelles (HSE). La DHG permet ainsi de calculer des postes en équivalent temps plein.

11. Heures d'enseignement par élève $(\mathrm{H} / \mathrm{E})$. Cet indicateur de taux d'encadrement dans le second degré rapporte les heures d'enseignement, dispensées hebdomadairement, aux nombres d'élèves. Il dépend notamment des horaires des programmes et des tailles des divisions (classes).

12. Définie en fonction du statut des enseignants par le décret $n^{\circ}$ 2014-940 du 20 août 2014, complété par la circulaire n²015-057 du 29 avril 2015. 
- Calcul de la « DHG théorique avec les options obligatoires » nommée DGH2 (Langues vivantes, Travaux Pratiques... à choisir parmi un panel de modules),

- Calcul de la «DHG théorique avec les options facultatives existantes » nommée DHG3.

L'application de ces trois calculs sur les Lycées de l'Académie témoin, EPPE donc, a confirmé l'effet « structure existante » ou poids du passé et des habitudes installées difficiles à modifier. Cet effet était renforcé par un effet contexte qui va de la personnalité du chef d'établissement, de son influence au-delà de l'établissement, au climat social dans l'établissement en passant par l'influence des politiques locaux, le tout jouant bien souvent en faveur d'un statu quo dans les moyens. Comme dans toute innovation managériale, l'introduction de cette modalité de pilotage de la performance fait que les logiques différentes des acteurs (Boitier et Rivière 2016) conduisent ces derniers aux jeux de pouvoir (Crozier et Friedberg 1977).

Face à cette situation, l'équipe de direction de l'Académie a décidé de mettre en place de nouvelles modalités d'attribution des moyens en heures en regroupant le dialogue stratégique et le dialogue de gestion, avec pour objectif d'assurer un niveau de dotation à DHG2 pour tous les établissements et de discuter ensuite de la marge d'autonomie (DHG3 - DHG2). Un pilotage des EPLE sur le territoire sur la base d'une certaine équité selon les performances constatées était le principe directeur de base de cette nouvelle modalité d'attribution des moyens en heures.

À ces moments de dialogue, la présence, face aux équipes de direction des EPLE, des DASEN ${ }^{13}$, des inspecteurs, de la Collectivité locale et des services académiques était supposée garantir l'efficacité du dispositif. Les acteurs disposant d'un levier d'action étaient donc présents et faisaient valoir celui-ci via le couple « responsabilités-résultats » inhérent à la méthode OVAR ${ }^{\circledR}$. La discussion reposait sur les indicateurs

13. Directeur Académique des Services de I'Éducation Nationale (DASEN), adjoint du Recteur responsable de l'attribution des moyens dans les départements de l'Académie. 
APAE $^{14}$, sur le projet d'établissement, sur le Tableau de Répartition des Moyens (TRM) et sur la DHG. L'objectif était d'aboutir à l'ajustement de certaines variables à partir d'indicateurs partagés et de responsabilités établies. Le contrat d'objectif issu de ces « dialogues de gestion de la performance » engageait toutes les parties prenantes. Un dispositif d'auto-évaluation et un accompagnement par les services académiques et les corps d'inspection complétaient le dispositif notamment à des fins d'amélioration. Les processus à l'œuvre pouvaient être discutés à l'aune du degré effectif d'atteinte des objectifs. Les discussions sur les variables d'action commençaient à se développer en parallèle des responsabilités dans le cadre des projets d'établissement.

\subsubsection{Une expérimentation innovante devenue un acte manqué!}

Le dispositif a été soutenu par l'implication de deux Recteurs successifs qui ont souhaité sa continuité, convaincus par les gestionnaires de la pertinence de l'expérimentation. Mais il n'a pas résisté à un troisième changement de Recteur qui a préféré mobiliser sur un changement d'échelle de l'organisation vers des réseaux d'établissements (en lieu et place des bassins de formation). L'expérimentation n'a pas été reconduite en l'état pour une seconde raison : elle a été jugée « trop technocratique » par l'administration centrale (DEPP $\left.{ }^{15}\right)$, empêchant, comme souvent, une véritable évaluation du dispositif.

L'expérimentation s'est heurtée in fine à une forme d'absence de réelle volonté politique de responsabiliser les établissements peu vertueux sur la base d'objectifs définis avec les parties prenantes, mais aussi probablement à un problème de « quantification de buts crédibles convergents entre eux ${ }^{16} »$ (Bouquin 2005, p. 126). En effet, comme le souligne Thélot (2008), d'une part, « il est beaucoup plus facile de

14. Aide au Pilotage et à l'Auto-évaluation des Établissements (APAE). C'est un outil de pilotage destiné aux chefs d'établissement qui a remplacé les Indicateurs de Pilotage de I'Enseignement Secondaire (IPES). Les données portent sur cinq années scolaires et permettent de situer l'établissement par rapport au département, à l'Académie et au niveau national. Il est également possible de paramétrer un tableau de bord personnalisé, notamment pour avoir une comparaison avec d'autres EPLE ou des niveaux agrégés (Source : site de l'ESEN).

15. Direction de l'Évaluation, de la Prospective et de la Prévision (DEPP) au sein du Secrétariat Général du Ministère.

16. Entre des objectifs politiques et gestionnaires dans la situation décrite. 
contrôler, d'évaluer et de recommander que d'agir, de conduire et de réformer » et d'autre part, " il faudrait que les recommandations qui en émanent ne soient pas trop difficiles à appliquer, pas trop contestables, pointues ou générales et réalistes » (p. 107).

La frilosité politique l'a, semble-t-il, emporté sur l'intention gestionnaire d'un pilotage innovant de la performance des EPLE. L'identification des responsabilités permise par la méthode semblait plus stigmatisante pour certains acteurs que responsabilisante à des fins d'amélioration des performances. Ces acteurs ont emporté la décision auprès du Recteur en faveur du retour à l'ancienne méthode qui sépare la négociation des objectifs (niveau politique) et celle des moyens (niveau gestionnaire). Les rapports entre politique et gestionnaire ont, semble-t-il, perturbé la mise en œuvre de la méthode, parce que les impacts politiques (éviter une stigmatisation de certains EPLE, créer du mécontentement par une baisse de moyens, publiciser le souci d'égalité du service public) n'étaient plus alignés sur les impacts gestionnaires (responsabiliser les acteurs dans les EPLE, faire jouer des variables d'actions identifiées, contribuer aux objectifs de politique publique).

L'innovation instrumentale oscillerait entre politisation des outils de gestion et un management public qui résiste à la nécessaire contingence par son « universalisme » où le principe d'égalité du service public est confondu avec une nécessaire équité territoriale. II existe en effet, au niveau académique, le projet académique qui, outre une fonction institutionnelle, a également une fonction managériale en intégrant le contrôle des réalisations et la mesure des impacts ou des extrants. La phase de construction du projet académique est cruciale car elle permet de confronter les aspirations (ou les devoirs) du politique et les exigences du gestionnaire (Jougleux et Pellegrin 2012).

\section{Discussion/Conclusion}

En s'appuyant sur l'analyse de l'étude de cas, nous avons cherché à comprendre comment l'interaction des logiques gestionnaire et politique peut-elle freiner la mise en œuvre d'un outil de pilotage de la performance? Malgré sa montée en puissance au sein des organisations publiques, le « managérialisme » semble fragile au regard d'une rationalité politique quelque peu erratique. Au-delà de ce constat, on 
peut se demander en effet si le NPM, s'inspirant des outils et des rationalités en jeu dans le secteur privé, n'est finalement pas un élément de rhétorique politique dans laquelle le "managérialisme », à travers les outils de gestion, serait instrumentalisé par le discours politique de façon asymétrique. II viendrait en effet appuyer de cette manière les " conclusions du contrôle », le discours justifiant des choix de politiques éducatives locales selon les résultats. Le management opérationnel et ses innovations seraient instrumentalisés par le politique au gré de son orientation générale et des jeux d'acteurs politiques. La politisation des outils de gestion, comme la comptabilité de gestion ou les tableaux de bord, a déjà été mise en évidence par Fabre (2014) dans le cadre de l'allocation des ressources au sein des collectivités locales. Ce qui conduirait, par exemple, au risque "d'extraire certains indicateurs en vue de la communication politique et de retenir un indicateur parmi d'autres, plus facilement compréhensible et correspondant à une priorité politique »(Brocas et Von Lennep 2010, p. 7).

II y aurait des établissements territorialement vertueux selon la chaîne " objectifs-variables d'action-responsabilités » et d'autres établissements que le contrôle « jugement »- au sens d'Hofstede (1981) est " censé responsabiliser » à la recherche de leviers de performance (qui sont à imaginer localement dans les établissements mêmes). La démarche initiale consistant à co-déterminer a) les objectifs opérationnels, b) les variables d'action, c) les responsabilités, ne se contente pas de poser les bases d'une correction chiffrée. C'est un outil remarquable de correction partagée des représentations du monde éducatif en question. Elle est, en soi, une action correctrice de l'inadaptation progressive des esprits et des faits. Naturellement, le partage de ce diagnostic devra être suivi d'effets progressifs sur une durée pluriannuelle, mais selon une démarche qui n'a pas lieu d'être automatique.

Les trois dotations horaires globalisées calculées et les écarts qui en résultent, ne sont pas un simple constat justifiant la mise en place de modalités d'attribution uniformes. Le résultat dessine en fait une carte du slack organisationnel (Cyert et March 1956) à partir de laquelle va pouvoir être mise en place, par le Recteur connaissant bien son terrain, une stratégie progressive. Ce qu'une telle carte révèle d'abord, c'est un ensemble de poches de slack qui ont perdu leurs raisons d'être, en totalité ou en partie, parce que la personne qui l'avait portée est partie ailleurs, quelle n'a plus vraiment d'implication opérationnelle. Cette 
première source de réallocation peut être mise en œuvre de façon relativement indolore, au profit des établissements les plus mal dotés. Cela peut éventuellement suffire pour la première année. Le Recteur va utiliser ensuite sa connaissance des chefs d'établissement et des quelques personnes qui comptent dans chacun : caractère, base de pouvoir à l'intérieur, appuis avérés dans l'Administration centrale et dans le monde politique local ou national, pour mettre en œuvre sa deuxième vague : contributions volontaires ou plus ou moins fortement sollicitées, sur la base de l'état des lieux connu de tous, et recalculé et republié au terme de la première année, et, dans quelques cas peut-être, affectation à un établissement défavorisé d'un personnel dynamique.

Notre étude confirme des études antérieures (Bryson et Roering 1988 ; Charih et Paquin 1993 ; Maurel 2006) qui mettent en lumière I'importance des groupes et individus en charge du management technique et gestionnaire dans les processus de changement au sein des organisations et administrations publiques. Mais, elle montre aussi que le politique est loin d'obéir à la rationalité managériale de performance et de gouvernance territoriales, dans notre cas du moins. L'adoption d'outils de pilotage en management public n'est, en outre, pas acquise définitivement et ne peut être réduite à une simple question de légitimité institutionnelle (Carassus et al. 2013) et sa non-diffusion à une simple désinstitutionalisation. Des facteurs complexes parfois dépendants sont à l'œuvre (instrumentalisation des outils par les politiques, notamment). Ainsi, les facteurs constituant des freins et des obstacles qui entravent les processus d'innovation dans le secteur public, qu'ils soient de type culturel ou organisationnel ou encore qu'ils soient liés à la nature politique des processus de décision, doivent être étudiés. L'idéal serait de combiner de tels facteurs dans une étude intégrée.

Notre étude montre que la relation gestionnaire(s)-politique, qui était un facteur favorable lors de la mise en œuvre de l'outil de pilotage décrit ici est devenue un facteur bloquant parce que les priorités politiques avaient changé. On est au cœur, ici, d'un problème de politique de la nation. Et, on peut se demander si la démarche de contrôle de gestion est vraiment dépourvue de moyens à cet égard. 


\section{Bibliographie}

Ahrens, T., Chapman, C. (2006). Doing qualitative weld research in management accounting: Positioning data to contribute to theory. Accounting, Organizations and Society 31 (8): 819-841.

Alcouffe, S., Boitier, M., Rivière, A. et Villesèque-Dubus, F. (2013). Contrôle de gestion sur mesure. Paris : Dunod.

Berland, N., De Rongé, Y. (2016). Contrôle de gestion. Perspectives stratégiques et managériales. Paris: Pearson.

Bessire, D., Fabre, P. (2011). Enjeux et limites du pilotage par les indicateurs en management public, l'exemple de la recherche en Sciences de Gestion. $32^{\text {e }}$ Congrès annuel de I'Association Francophone de Comptabilité, Montpellier.

Boitier, M., Rivière, A. (2016). Les systèmes de contrôle de gestion, vecteurs d'une logique gestionnaire : changement institutionnel et conflits de logiques à l'université. Comptabilité - Contrôle - Audit 22 (3) : 47-79.

Bollecker, M. (2013). Pourquoi le contrôle de gestion à I'Université est un échec ? Politiques et Management Public 30 (2) : 221-239

Bollecker, M. (2016). L'adoption de la comptabilité de gestion à l'université : la présence de clivages internes dans un contexte de demandes institutionnelles contradictoires. Comptabilité - Contrôle - Audit 22 (2) : 109-138.

Brocas, A.-M., Von Lennp, F. (2010). Contrepoint : de l'utilité, (et des limites) du recours aux indicateurs pour le pilotage des politiques publiques. RFAS $1-2: 149-160$.

Bouquin, H. (Dir., 2005), Les grands auteurs en contrôle de gestion. Paris : Éditions Management et Société.

Bryson, J. M., Roering, W. D. (1988). Initiation of strategic planning by governments. Public Administration Review 48 (6) : 995-1004.

Carassus, D., Favoreu, C., Gardey, D., Maurel, C. (2014). Les déterminants de l'adoption et de la mise en œuvre d'un management par la performance : application aux collectivités locales françaises Finance - Contrôle - Stratégie 17 (1) : 1-24.

Carassus, D., Favoreu, C., Gardey, D. (2013). Factors that Determine or Influence Managerial Innovation in Public Contexts: The Case of Local Performance Management. Public Organization Review 13 (1): 245-266.

Chanal, V., Lesca, H., Martinet, A. (2015). Vers une ingénierie de la recherche en sciences de gestion. Revue française de gestion 253 (8) : 213-229. 
Charih, M., Paquin, M. (1993). La planification stratégique à Ottawa et Québec : une comparaison de quelques ministères. Canadian Public Administration 36 (2) : 175-189.

Crozier, M., Friedberg, E. (1977). L'acteur et le système. Paris : Seuil.

Cyert, R., March, J. (1956). Organizational factors in the theory of oligopoly. The Quarterly Journal of Economics 70 (1): 44-64.

Damanpour, F., Schneider, M. (2008). Characteristics of Innovation and Innovation Adoption in Public Organizations: Assessing the Role of Managers. Journal of Public Administration Research and Theory 19 (3): 495-522.

Dreveton, B., Lande, E., Portal, M. (2012). Construire un outil de comptabilité de gestion au sein d'une université. Retour sur un acte manqué. Management \& Avenir 54 (4) : 126-144.

Dreveton, B. (2014). Étude du rôle des objets au cours du processus d'instrumentation des activités publiques. Comptabilité - Contrôle - Audit 20 (1) : 43-66.

Dreveton, B. (2017). Les outils du contrôle de gestion : des vecteurs de valeurs pour l'organisation publique ? Comptabilité - Contrôle - Audit 23 (3) : 9-28

Ducrocq, C., Gervais, M. (2013). Le coût complet par diplôme à l'université : quel apport vu les problèmes de mesure ? Finance - Contrôle - Stratégie 16 (4) : 1-29.

Eyraud, C. (2013). Mesurer l'action publique par des indicateurs. Systèmes de valeurs et jeux de pouvoir. In Chiapello, E., Gilbert, P. (coord.). Sociologie des outils de gestion (183-196). Paris : Éditions La Découverte

Fabre, P. (2014). Contrôle de gestion et management public : quel retour d'expérience pour les entreprises. In Bollecker, M., Naro, G. (coord.). Le contrôle de gestion aujourd'hui. Débats, controverses et perspectives (5973). Paris : Vuibert.

Gould-Williams, J. (2004). The Effects of High Commitment HRM Practices on Employee Attitude: The Views of Public Sector Workers. Public Administration 82 (1): 63-82.

Hofstede, G. (1981). Management control of public and not-for-profit activities. Accounting, Organizations and Society 6 (3) : 193-211.

Inspection Générale de l'Éducation Nationale (2007). Le pilotage du système éducatif dans les académies à l'épreuve de la LOLF, rapport conjoint n²006-85, Ministère de l'Éducation Nationale.

Jougleux, M., Pellegrin, N. (2012). Quel pilotage pour le système éducatif des académies ? Politiques et Management Public 29 (3) : 369-392. 
Kurunmakï, L. (2009). Management accounting, Economic Reasoning and the New Public Management Reforms. In Chapman, C., Hopwood, A., Schelds, M. (Eds). Handbook of Management Accounting Research: 13711383.

Lalonde, J.-F. (2013). La participation observante en sciences de la gestion : plaidoyer pour une utilisation accrue. Recherches Qualitatives 32 (2) : 13-32.

Löning, H., Selmer, C. (2011). Les outils de pilotage : de la mesure au management de la performance. In Berland, N., Simon, F.-X. (coord). Le contrôle de gestion en mouvement (107-123). Paris : Éditions d'Organisation.

Lorino, Ph. (1999). La recherche de la valeur perdue : construire les processus créateurs de valeur dans le secteur public. Politiques et Management Public 17 (2) : 21-33.

Lorino, Ph. (1998). Méthodes et pratiques de la performance. Le guide du pilotage. Paris : Éditions d'Organisation.

Maurel, L.-C. (2006). Étude d'un changement organisationnel dans de grandes collectivités territoriales : I'évolution de la fonction contrôle de gestion. Finance - Contrôle - Stratégie 9 (3) : 105-134.

Moynihan, D.-P. (2006). Managing for Results in State Government: Evaluating a Decade of Reform. Public Administration Review 66 (1): 78-90.

Porter, M. (1986). L'avantage concurrentiel. Paris : InterEditions.

Selmer, C. (2005). Un outil d'analyse et d'aide à la décision. In Balantzian, G. (Ed.), Tableaux de bord (17-43). Paris : Éditions d'Organisation.

Stake, R.E. (1994). Case studies. In Denzin N. K., Linclon, Y. S (Eds), Handbook of qualitative research (236-247).

Thelot, C. (2008). La principale faiblesse de l'évaluation et du contrôle de l'école en France. Revue internationale d'éducation de Sèvres, $\mathrm{n}^{\circ} 48$ : 97-108.

Walker, R. (2006). Innovation Type and Diffusion: An Empirical Analysis of local Government. Public Administration 84 (2): 311-335. 